\title{
Identification and correction of antisymmetric field components in combined function magnets
}

\author{
M. Seidl* \\ Institut für Kernphysik, Universität Mainz, D-55099 Mainz, Germany
}

(Received 23 October 2001; published 12 June 2002)

\begin{abstract}
This paper investigates the identification and correction of antisymmetric field components in combined function magnets. A measurement procedure is developed to identify small field components on the order of $10^{-3}$ in the plane of the reference path. Furthermore, a method is proposed to compensate them by surface correction coils on the poles of the magnet. As an example, the measurement and compensation of antisymmetric field components in a $90^{\circ}$ bending magnet of the double sided microtron HDSM at the MAMI-C electron scattering facility is simulated with the help of the electromagnetic design code TOSCA.
\end{abstract}

DOI: 10.1103/PhysRevSTAB.5.062402

PACS numbers: 29.20.-c, 41.85.Lc

\section{INTRODUCTION}

Field components in the plane of the reference path lead to a coupling between the horizontal, vertical, and longitudinal phase spaces in combined function magnets $[1,2]$. They are typically on the order of $10^{-3}$ if the magnet is carefully designed and manufactured. To the author's knowledge, they were hitherto neglected in the identification and correction of field errors when hall probes were the only option for field measurement [3-5]. However, the construction of more advanced combined function magnets such as the $90^{\circ}$ dipoles of the double sided microtron HDSM at MAMI-C (see Sec. IV) makes it necessary to compensate even those small field errors over an area greater than $6 \mathrm{~m}^{2}$.

In Sec. II, a procedure is proposed to experimentally determine antisymmetric field components. A method to compensate them by surface correction coils is given in Sec. III. Finally, the identification and compensation of antisymmetric field components in a $90^{\circ} \mathrm{HDSM}$ magnet is simulated with the help of TOSCA [6] in Sec. V.

If $\vec{j}(\vec{q})$ is the current density at location $\vec{q}$, the vector potential $\vec{A}$ is [7]

$$
\vec{A}(\vec{q})=\frac{1}{4 \pi \epsilon_{0} c^{2}} \int d \vec{q}^{\prime} \frac{\vec{j}\left(\vec{q}^{\prime}\right)}{\left|\vec{q}-\vec{q}^{\prime}\right|} .
$$

The magnetization vector within the magnet is $\vec{M}$. It is equivalent to the following current density $\vec{j}_{M}$ :

$$
\vec{j}_{M}=\nabla \times \vec{M} \text {. }
$$

Inserting $\vec{j}_{M}$ into Eq. (1) and integrating over the volume $V$ and surface $S$ of the magnet yields [7]

$$
\begin{aligned}
\vec{A}_{M}(\vec{q})= & \frac{1}{4 \pi \epsilon_{0} c^{2}}\left(\int_{V} d V\left(\vec{q}^{\prime}\right) \frac{\nabla^{\prime} \times \vec{M}\left(\vec{q}^{\prime}\right)}{\left|\vec{q}-\vec{q}^{\prime}\right|}\right. \\
& \left.+\oint_{S} d S\left(\vec{q}^{\prime}\right) \frac{\vec{M}\left(\vec{q}^{\prime}\right) \times \vec{n}^{\prime}\left(\vec{q}^{\prime}\right)}{\left|\vec{q}-\vec{q}^{\prime}\right|}\right),
\end{aligned}
$$

\footnotetext{
*Present address: TÜV Süddeutschland Bau und Betrieb GmbH, D-80686 München, Germany.
}

where $\vec{n}^{\prime}$ is the surface normal vector of $S$. In the following, it is assumed that the magnetization $\vec{M}$ is homogeneous in $V$, or that the gap of the magnet is much smaller than $V$. Then the volume integral in Eq. (3) can be considered as a constant. Assume that the magnetization $\vec{M}_{e}$ on the surface of the real magnet is somehow known. To bring it in accordance with the design calculation for $\vec{M}$, a correction current $\delta \vec{j}$ is needed:

$$
\delta \vec{j}=\nabla \times\left(\vec{M}-\vec{M}_{e}\right) .
$$

In magnetostatics we have

$$
\nabla \times\left(\vec{B}-\frac{1}{\epsilon_{0} c^{2}} \vec{M}\right)=0,
$$

and thus

$$
\delta \vec{j}=\epsilon_{0} c^{2} \nabla \times\left(\vec{B}-\vec{B}_{e}\right) .
$$

Therefore the magnetic field $\vec{B}_{e}$ at the surface of the poles of the magnet has to be determined experimentally. From a comparison of $\vec{B}_{e}$ with the design calculation for $\vec{B}$, surface correction coils can be constructed as shown in Eq. (6). For (anti)symmetrical field errors, the correction current $\delta \vec{j}$ is (anti)symmetrical with respect to the midplane. The corresponding field components are perpendicular to the midplane in the case of symmetry and are parallel to it in the case of antisymmetry.

\section{MEASUREMENT OF ANTISYMMETRIC FIELD COMPONENTS}

Assume that the reference path lies in the $x-z$ plane, which is also the median plane of the magnet, and that the main field component is parallel to the $y$ axis. It is not experimentally feasible to directly measure the $B_{x}$ and $B_{z}$ components of the field as a function of $(x, z)$ in the presence of a $B_{y}$ component that is approximately $10^{3}$ times larger. Therefore, the procedure proposed by Wind [8] is not applicable. Harmonic coils [9] would be another option if the field errors are expected to be invariant with $x$ or $z$, which is not assumed here. 
The following procedure circumvents a direct measurement of $B_{x}$ and $B_{z}$. In the gap of the magnet, Maxwell's equations yield for the magnetostatic case:

$$
\nabla \cdot \vec{B}=0,
$$

and

$$
\nabla \times \vec{B}=0 .
$$

Differentiation of Eq. (7) with respect to $x$ and $z$ gives

$$
\frac{\partial^{2} B_{x}}{\partial x^{2}}+\frac{\partial^{2} B_{z}}{\partial z \partial x}=-\frac{\partial^{2} B_{y}}{\partial y \partial x},
$$

and

$$
\frac{\partial^{2} B_{z}}{\partial z^{2}}+\frac{\partial^{2} B_{x}}{\partial z \partial x}=-\frac{\partial^{2} B_{y}}{\partial y \partial z},
$$

respectively. From Eq. (8), it follows that

$$
\frac{\partial B_{x}}{\partial z}=\frac{\partial B_{z}}{\partial x} .
$$

Inserting this relation into Eqs. (9) and (10) results in

$$
\frac{\partial^{2} B_{x}}{\partial x^{2}}+\frac{\partial^{2} B_{x}}{\partial z^{2}}=-\frac{\partial^{2} B_{y}}{\partial y \partial x},
$$

and

$$
\frac{\partial^{2} B_{z}}{\partial x^{2}}+\frac{\partial^{2} B_{z}}{\partial z^{2}}=-\frac{\partial^{2} B_{y}}{\partial y \partial z} .
$$

The right-hand sides of Eqs. (12) and (13) can be determined by a measurement of $B_{y}$ below and above the median plane of the magnet. If $h_{y} / 2$ is the distance from the median plane, then

$$
\frac{\partial B_{y}}{\partial y}(x, 0, z) \approx \frac{1}{h_{y}}\left[B_{y}\left(x, \frac{h_{y}}{2}, z\right)-B_{y}\left(x,-\frac{h_{y}}{2}, z\right)\right] .
$$

Thus if $B_{y}$ is measured on a grid with spacing $h_{x}$ and $h_{z}$, one can determine $\partial_{y x} B_{y}$ and $\partial_{y z} B_{y}$ with the help of Eq. (14) as follows:

$$
\frac{\partial^{2} B_{y}}{\partial y \partial x} \approx \frac{1}{h_{y} h_{x}}\left[B_{y}\left(x+\frac{h_{x}}{2}, \frac{h_{y}}{2}, z\right)-B_{y}\left(x+\frac{h_{x}}{2},-\frac{h_{y}}{2}, z\right)+B_{y}\left(x-\frac{h_{x}}{2},-\frac{h_{y}}{2}, z\right)-B_{y}\left(x-\frac{h_{x}}{2}, \frac{h_{y}}{2}, z\right)\right],
$$

and

$$
\frac{\partial^{2} B_{y}}{\partial y \partial z} \approx \frac{1}{h_{y} h_{z}}\left[B_{y}\left(x, \frac{h_{y}}{2}, z+\frac{h_{z}}{2}\right)-B_{y}\left(x,-\frac{h_{y}}{2}, z+\frac{h_{z}}{2}\right)+B_{y}\left(x,-\frac{h_{y}}{2}, z-\frac{h_{z}}{2}\right)-B_{y}\left(x, \frac{h_{y}}{2}, z-\frac{h_{z}}{2}\right)\right] .
$$

In mathematical terms, both Eqs. (12) and (13) are partial differential equations of the Dirichlet type. Assume that $B_{x}$ and $B_{z}$ are zero at the boundary of the measurement region, and that in the median plane $\partial_{y x} B_{y}$ and $\partial_{y z} B_{y}$ are known from measurement. Then each equation has a unique solution for $B_{x}$ and $B_{z}$ in the median plane. In general, both field components can be determined by numerical integrations of Eqs. (12) and (13). There exists an abundance of methods for this task [10]. Having solved both equations for $B_{x}$ and $B_{z}$ and additionally hav- ing measured $B_{y}$ in the median plane, all components of the magnetic field in the median plane of the magnet are determined.

\section{SURFACE CORRECTION COILS}

Now that $\vec{B}_{e}$ is known in the median plane of the real magnet, one can determine the magnetic field in the whole gap. In free space, $\vec{B}_{e}$ can be expressed as a gradient of a scalar potential $\Psi$ [7]. In the vicinity of a point $\left(x_{j}, 0, z_{i}\right)$ on the measurement grid, $\Psi$ can be expressed as follows:

$$
\begin{aligned}
\Psi= & a_{100}\left(x-x_{j}\right)+a_{010} y+a_{001}\left(z-z_{i}\right)+\frac{1}{2} a_{200}\left(x-x_{j}\right)^{2}+\frac{1}{2} a_{020}(y)^{2}+\frac{1}{2} a_{002}\left(z-z_{i}\right)^{2} \\
& +a_{110}\left(x-x_{j}\right) y+a_{011} y\left(z-z_{i}\right)+a_{101}\left(x-x_{j}\right)\left(z-z_{i}\right)+\cdots
\end{aligned}
$$

All coefficients are now known:

$$
\begin{aligned}
& a_{100}=B_{x}\left(x_{j}, 0, z_{i}\right), \\
& a_{010}=B_{y}\left(x_{j}, 0, z_{i}\right), \\
& a_{001}=B_{z}\left(x_{j}, 0, z_{i}\right),
\end{aligned}
$$


and

$$
\begin{aligned}
& a_{200}=\frac{\partial B_{x}}{\partial x}\left(x_{j}, 0, z_{i}\right) \approx \frac{1}{h_{x}}\left[B_{x}\left(x_{j}+h_{x}, 0, z_{i}\right)-B_{x}\left(x_{j}, 0, z_{i}\right)\right], \\
& a_{020}=-a_{200}-a_{002}, \\
& a_{002}=\frac{\partial B_{z}}{\partial z}\left(x_{j}, 0, z_{i}\right) \approx \frac{1}{h_{z}}\left[B_{z}\left(x_{j}, 0, z_{i}+h_{z}\right)-B_{z}\left(x_{j}, 0, z_{i}\right)\right],
\end{aligned}
$$

and

$$
\begin{aligned}
& a_{101}=\frac{\partial B_{x}}{\partial z}\left(x_{j}, 0, z_{i}\right) \approx \frac{1}{h_{z}}\left[B_{x}\left(x_{j}, 0, z_{i}+h_{z}\right)-B_{x}\left(x_{j}, 0, z_{i}\right)\right], \\
& a_{110}=\frac{\partial B_{y}}{\partial x}\left(x_{j}, 0, z_{i}\right) \approx \frac{1}{h_{x}}\left[B_{y}\left(x_{j}+h_{x}, 0, z_{i}\right)-B_{y}\left(x_{j}, 0, z_{i}\right)\right], \\
& a_{011}=\frac{\partial B_{y}}{\partial z}\left(x_{j}, 0, z_{i}\right) \approx \frac{1}{h_{z}}\left[B_{y}\left(x_{j}, 0, z_{i}+h_{z}\right)-B_{y}\left(x_{j}, 0, z_{i}\right)\right] .
\end{aligned}
$$

Using this approach, $\Psi$ and thus $\vec{B}_{e}$ can be determined at the poles of the magnet. Neglecting a possible curvature of the poles, the surface current density $\vec{\sigma}$ at the upper pole can be calculated from Eq. (6)

$$
\begin{aligned}
& \sigma_{x}=+\epsilon_{0} c^{2}\left(B_{z}-B_{z, e}\right), \\
& \sigma_{z}=-\epsilon_{0} c^{2}\left(B_{x}-B_{x, e}\right),
\end{aligned}
$$

if the relative permeability of the magnet is assumed to be infinite. The current density at the lower pole has a reversed sign. If a function $I(x, z)$ is defined as

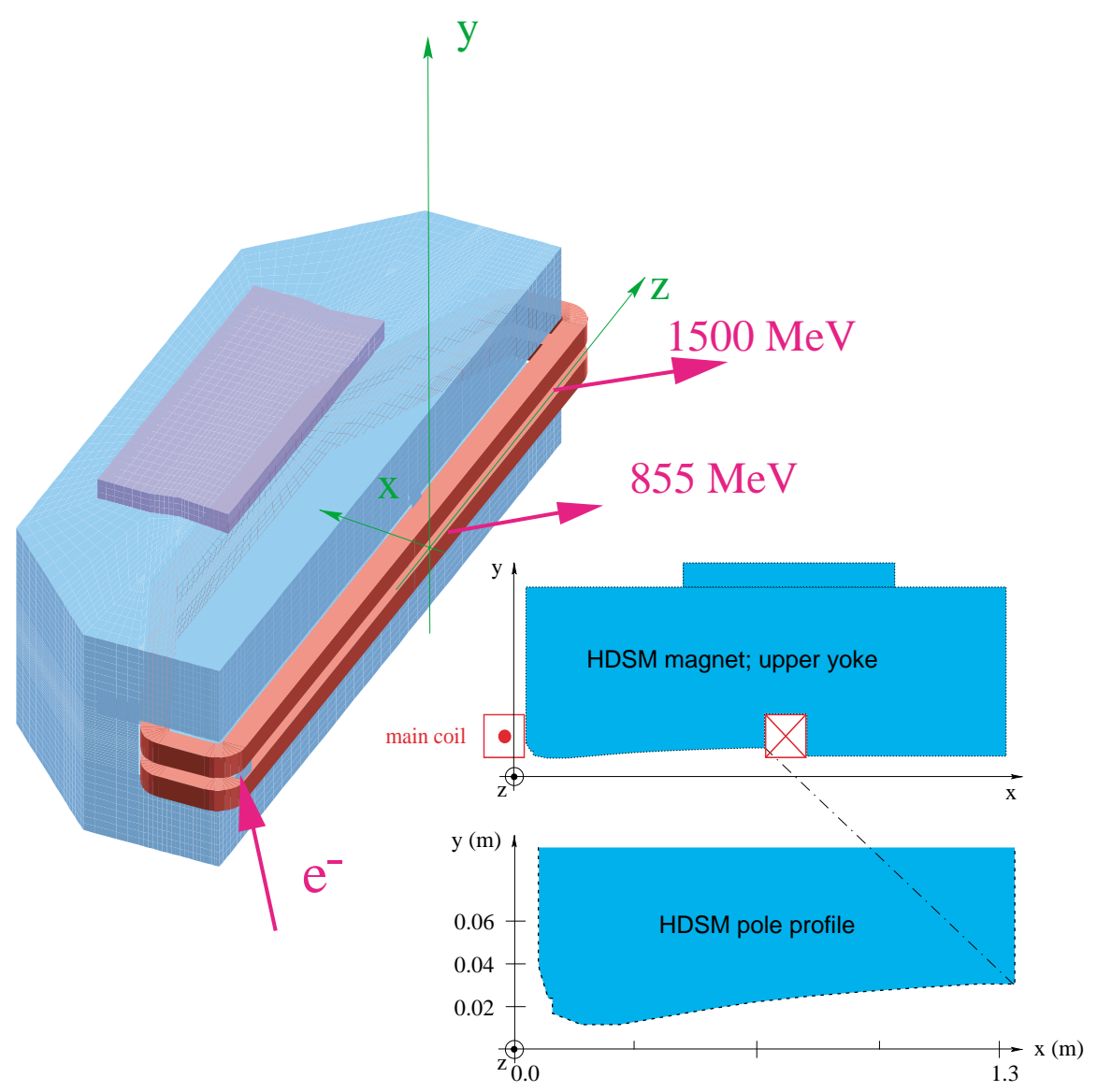

FIG. 1. (Color) Artist's view and pole profile of a $90^{\circ} \mathrm{HDSM}$ bending magnet. 


$$
I(x, z)=\int_{0}^{x} d s_{x} \sigma_{z}\left(s_{x}, z\right)-\int_{0}^{z} d s_{z} \sigma_{x}\left(0, s_{z}\right),
$$

one gets

$$
\frac{\partial I}{\partial x}=\sigma_{z} ; \quad \frac{\partial I}{\partial z}=-\sigma_{x}
$$

Therefore the surface correction coils have to run along the equipotential lines of $I$ with a current equal to the level distance $\Delta I$.

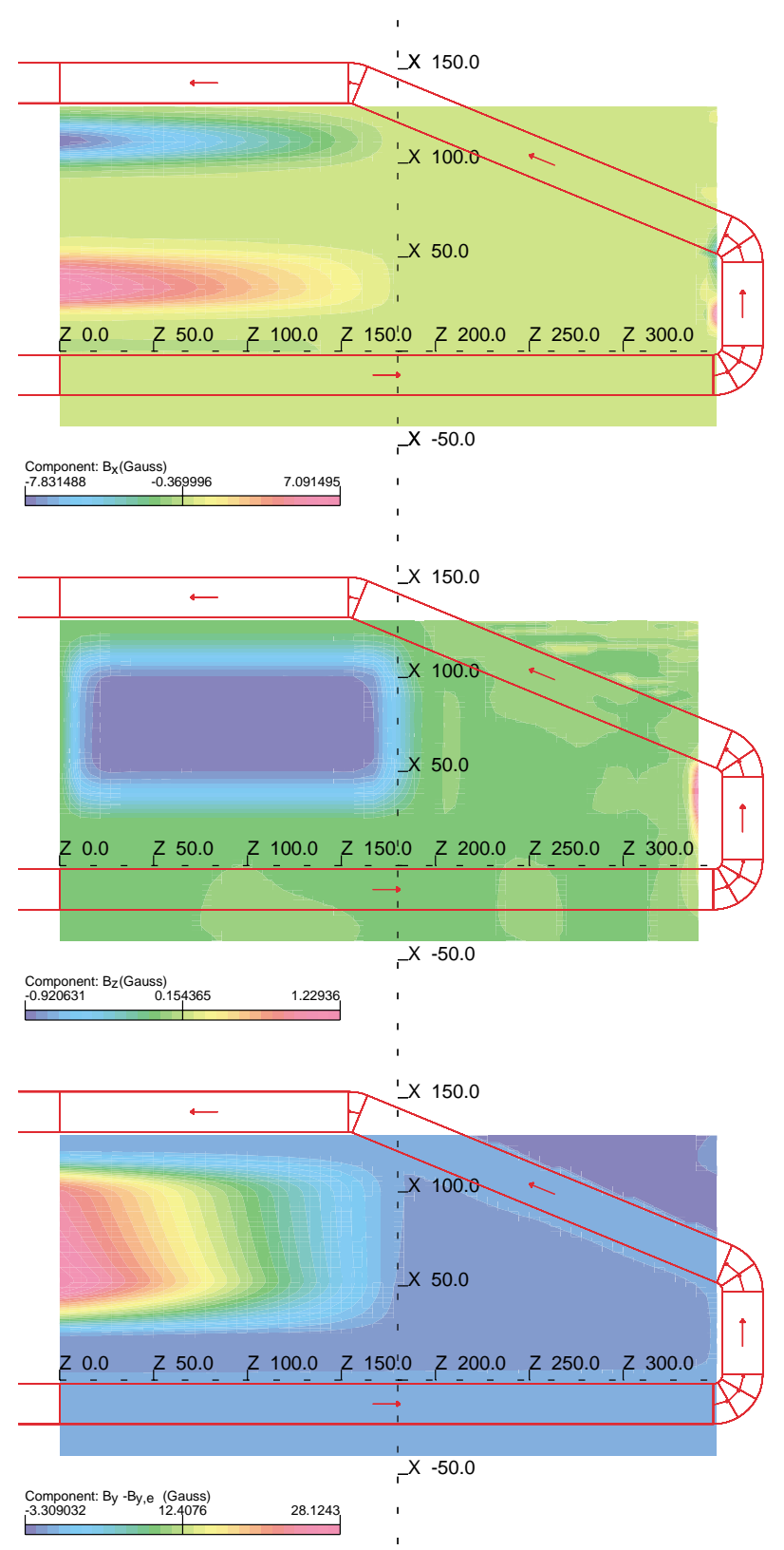

FIG. 2. (Color) Simulated field errors $B_{x}, B_{z}$, and $B_{y}-B_{y, e}$ in the median plane of a $90^{\circ}$ HDSM bending magnet with a slightly misaligned upper yoke.
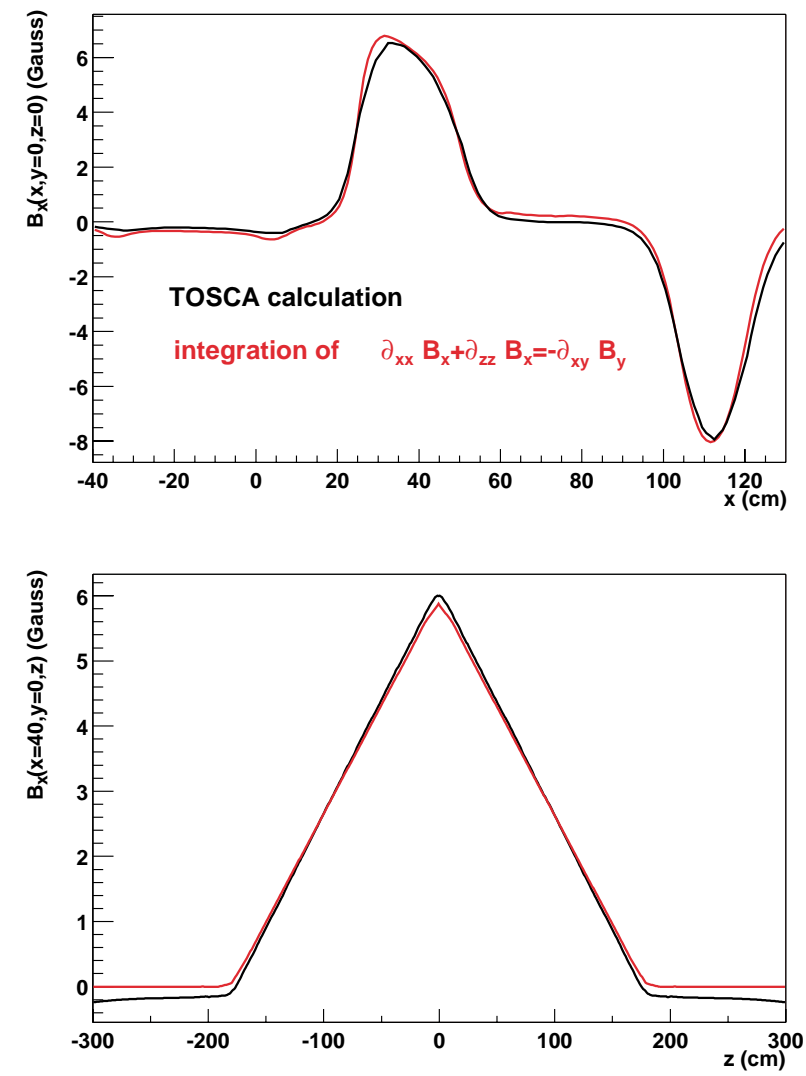

FIG. 3. (Color) TOSCA calculation of $B_{x}$ compared with the results of a simulated measurement of $\partial_{x y} B_{y}$ and subsequent integration of Eq. (12).

\section{MAMI-C}

At the MAMI [11] electron scattering facility, three racetrack microtrons are cascaded to provide a $\mathrm{cw}$ electron beam at $855 \mathrm{MeV}$. In a fourth stage, a double sided microtron HDSM is currently being built [12] to raise the beam energy to $1500 \mathrm{MeV}$ in 43 cycles. A 250 ton $90^{\circ}$ HDSM bending magnet [13] is shown in Fig. 1. Its field maximum for $B_{y}$ is $1.5 \mathrm{~T}$. To nullify the strong vertical defocusing forces of the fringe field for all beam energies between 855 and $1500 \mathrm{MeV}$, the magnets have an additional field gradient in the main field region [14]. Because of construction constraints on the sophisticated pole profile, it is expected that antisymmetric field components

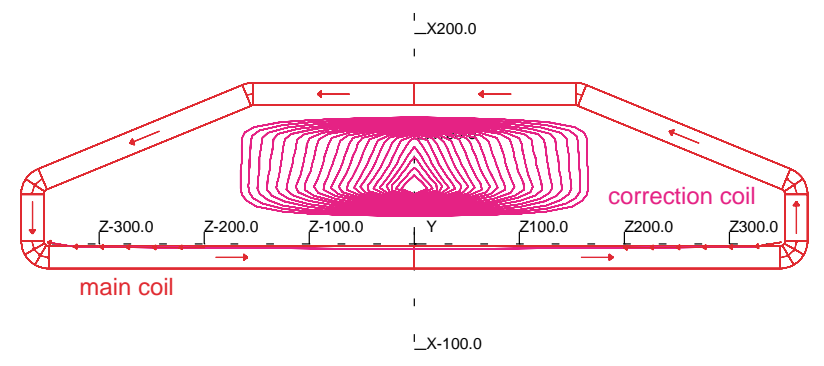

FIG. 4. (Color) Surface correction coils for the upper pole of a $90^{\circ}$ HDSM bending magnet. 


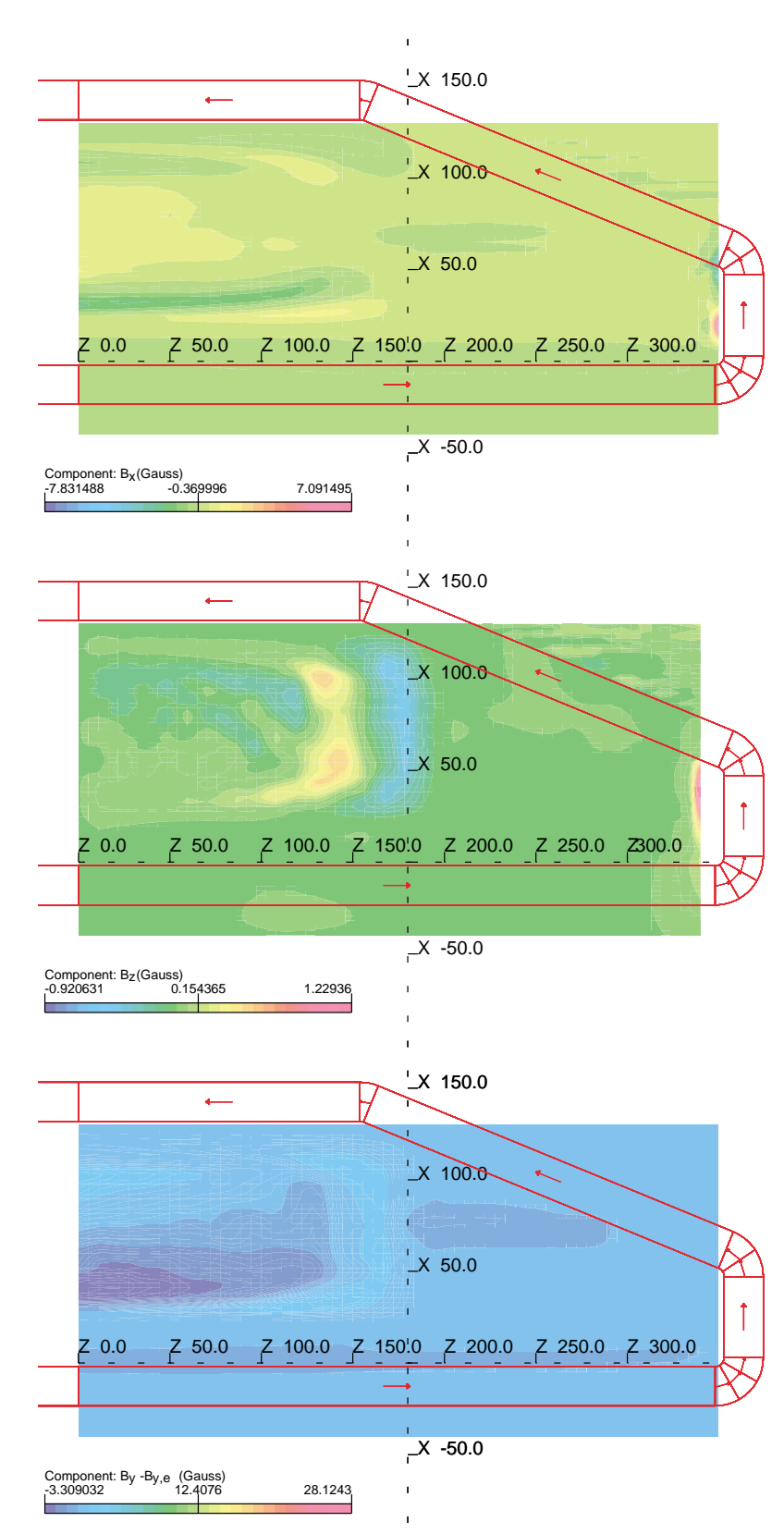

FIG. 5. (Color) Field components $B_{x}, B_{z}$, and $B_{y}-B_{y, e}$ in the midplane of a $90^{\circ} \mathrm{HDSM}$ bending magnet with surface correction coils to compensate field errors due to a slightly misaligned upper yoke.

in the median plane of the order of $10^{-3}$ have to be compensated.

\section{TOSCa SIMULATION}

To simulate antisymmetric field components in the median plane of a $90^{\circ}$ HDSM magnet, its upper yoke was not correctly aligned with respect to the lower yoke, but the maximum deviations of $0.3 \mathrm{~mm}$ were still within the manufacturing tolerance. The ensuing field errors were calculated with tOSCA and are shown in Fig. 2. From the Tosca calculation, the main field component $B_{y}$ in
TABLE I. Deviation of $B_{x}$ and $B_{z}$ from the TOSCA results $B_{x}$ T and $B_{z \mathrm{~T}}$ for various noise levels of $B_{y}$.

\begin{tabular}{ccccccc}
\hline \hline$\sigma_{B_{y}}($ Gauss $)$ & 0.0 & 0.5 & 1.0 & 2.0 & 4.0 & 8.0 \\
\hline$\sigma_{B_{x}-B_{x} \mathrm{~T}}$ (Gauss) & 0.05 & 0.15 & 0.26 & 0.47 & 0.91 & 1.78 \\
$\sigma_{B_{z}-B_{z \mathrm{~T}}}$ (Gauss) & 0.03 & 0.17 & 0.22 & 0.44 & 0.86 & 1.71 \\
\hline \hline
\end{tabular}

the median plane and at $h_{y} / 2=2 \mathrm{~cm}$ above and below the median plane was extracted on a grid with spacing $h_{x}=1 \mathrm{~cm}$ and $h_{z}=1 \mathrm{~cm}$. Then the field components $B_{x}$ and $B_{z}$ in the median plane were calculated according to Sec. II. In Fig. 3, the result for $B_{x}$ is compared with the original data given by TOSCA. They agree within 5\%. Finally, surface correction coils were calculated as described in Sec. III. On the lower pole, no correction coils were necessary in this example since the field errors were exclusively produced by the misaligned upper yoke. The surface correction coils for the upper pole are shown in Fig. 4. The magnetic field in the median plane was again calculated by TOSCA with the correction coils turned on. The result is shown in Fig. 5. In all cases, the field quality was improved by a factor of about 10 .

To estimate the robustness of the proposed procedure, assume in the following that $B_{x}$ is not a function of $z$ and $B_{z}$ is not a function of $x$. Then Eqs. (12) and (13) can be integrated with respect to $x$ and $z$, respectively:

$$
\delta B_{x} \approx-\frac{\delta x}{\delta y} \delta B_{y}
$$

and

$$
\delta B_{z} \approx-\frac{\delta z}{\delta y} \delta B_{y}
$$

Therefore the error in determining $B_{x}$ and $B_{z}$ is proportional to the measurement error of $B_{y}$. To verify this relationship numerically, Gaussian noise was added to the TOSCA data for $B_{y}$, and then $B_{x}$ and $B_{z}$ were recalculated according to Eqs. (12) and (13). Table I shows the sigma width of the ensuing deviation from the original TOSCA result for $B_{x}$ and $B_{z}$. The linear relationship as predicted by Eqs. (24) and (25) is well verified. For the used spacing $h_{y}=4 \mathrm{~cm}$ and $h_{x}=1, h_{z}=1 \mathrm{~cm}$, one would expect a slope of 1/4.0. The simulation yields $1 / 4.4$, which is probably due to the simplifying assumption leading to Eqs. (24) and (25).

\section{CONCLUSION}

In this paper, we demonstrated that it is possible to identify and compensate small antisymmetric field components on the order of $10^{-3}$ in combined function magnets. By measuring the main field components both in the median plane and below and above the median plane, one can derive all components of the magnetic field in the median plane. Thus the field in the whole gap of the magnet can be calculated from measurements. Surface correction coils 
can be constructed from a comparison of the experimentally determined magnetic field at the poles with the field of the design calculation.

\section{ACKNOWLEDGMENT}

I am indebted to K.-H. Kaiser and U. Ludwig-Mertin at Institut für Kernphysik, Universität Mainz for their cooperation. This work was supported by DFG (Graduiertenkolleg "Physik und Technik von Beschleunigern").

[1] D. Sagan and D. Rubin, Phys. Rev. ST Accel. Beams 2, 074001 (1999).

[2] H. Wiedemann, Particle Accelerator Physics (SpringerVerlag, Berlin, 1998), Vol. II.

[3] H. Herminghaus, K.-H. Kaiser, and U. Ludwig, Nucl. Instrum. Methods Phys. Res. 187, 103 (1981).

[4] L. R. P. Kassab and P. Gouffon, Phys. Rev. ST Accel. Beams 1, 012401 (1998).
[5] A. Morita, A. Noda, H. Tonguu, T. Shirai, and Y. Iwashita, in Proceedings of the 7th European Particle Accelerator Conference, edited by J. Pool and C. Petit (Institute of Physics, Bristol, U.K., 2000).

[6] TOSCA, software for Electromagnetic Design, Vector Fields Limited, Oxford, 1998.

[7] J. Jackson, Classical Electrodynamics (Wiley, New York, 1975).

[8] H. Wind, Nucl. Instrum. Methods 84, 117 (1970).

[9] A. Jain, U.S. Particle Accelerator School, Houston, 2001.

[10] A. Quarteroni and A. Valli, Numerical Approximation of Partial Differential Equations (Springer-Verlag, Berlin, 1994).

[11] H. Herminghaus, A. Feder, K.-H. Kaiser, W. Manz, and H. v. d. Schmitt, Nucl. Instrum. Methods 138, 1 (1976).

[12] DFG Collaborative Research Centre No. 443, 2001.

[13] U. Ludwig-Mertin, H. Euteneuer, K.-H. Kaiser, H.-J. Kreidel, and S. Ratschow, in Proceedings of the 6th European Particle Accelerator Conference, edited by J. Pool and C. Petit (Institute of Physics, Bristol, U.K., 1998).

[14] S. Ratschow, Ph.D. thesis, Institut für Kernphysik Universität Mainz, 2000. 